\title{
Upregulation of Salmonella-Induced IL-6 Production in Caco-2 Cells by PJ-34, PARP-1 Inhibitor: Involvement of PI3K, p38 MAPK, ERK, JNK, and NF- $\kappa$ B
}

\author{
Fu-Chen Huang \\ Department of Pediatrics, Chang Gung Memorial Hospital-Kaohsiung Medical Center, Chang Gung University College of Medicine, \\ 123, Ta-pei Road, Niao-sung Hsiang, Kaohsiung Hsien 833, Taiwan \\ Correspondence should be addressed to Fu-Chen Huang, huang817@adm.cgmh.org.tw
}

Received 29 July 2009; Revised 14 November 2009; Accepted 14 November 2009

Recommended by Vera L. Petricevich

\begin{abstract}
Following Salmonella invasion, intestinal epithelial cells release a distinct array of proinflammatory cytokines. Interleukin (IL)-6 produced by enterocytes may have anti-inflammatory and cell-protective effects, and may counteract some of the injurious effects of sepsis and endotoxemia. Recent studies in a variety of rodent models of experimental colitis by using PJ-34, a potent poly (ADP-ribose) polymerase-1 (PARP-1) inhibitor, support the concept that the marked beneficial effect of PJ-34 can be exploited to treat human inflammatory diseases. The present study was to investigate the effect of PJ-34 on Salmonella-induced enterocyte IL-6 production and its mechanisms. We found that PJ-34 enhanced Salmonella-induced IL-6 production in Caco- 2 cells, either secreted protein or mRNA expression. PJ-34 treatment enhanced the activity of NF- $\kappa$ B in Salmonella-infected Caco- 2 cells. Besides, the involvement of PJ-34 in up-regulating IL-6 production in S. typhimurium-infected Caco-2 cells might be also through the ERK but not p38 MAPK, JNK or PI3K/Akt pathways, as demonstrated by Western blot of phosphorylated ERK, p38, JNK and Akt proteins. It suggests that PJ-34 may exert its protective effect on intestinal epithelial cells against invasive Salmonella infection by up-regulating IL-6 production through ERK and NF- $\kappa$ B but not P38 MAPK, JNK or PI3K/Akt signal pathways.
\end{abstract}

Copyright (C) $2009 \mathrm{Fu}$-Chen Huang. This is an open access article distributed under the Creative Commons Attribution License, which permits unrestricted use, distribution, and reproduction in any medium, provided the original work is properly cited.

\section{Background}

Epithelial cells have been recognized as playing an important role in mucosal immunity through the expression of proinflammatory cytokines in response to microbial injury [1]. Among cytokines produced in the intestinal mucosa during sepsis and endotoxemia, interleukin (IL)-6 is particularly important because of its multiple significant biological effects [2]. Although commonly considered a proinflammatory cytokine [3], there is also evidence that IL-6 has important antiinflammatory properties and may exert protective effects in various tissues [4-6]. IL-6 plays an essential role in the intestinal barrier in L. monocytogenesinfected human intestinal epithelial cells $[7,8]$. Because of the multiple biological effects of IL-6, a better understanding of the molecular regulation of enterocyte IL- 6 production and methods to modulate IL-6 production in intestinal mucosa in infected enterocytes may have important clinical implications.
Over the last decade, a multitude of studies have verified the role of PARP-1 activation in a wide range of pathophysiologic conditions, such as arthritis, asthma, inflammatory bowel disease, lung inflammation, multiple organ failure, and septic shock [9]. The marked beneficial effect of PARP inhibitors in these animal models of various diseases also suggests that PARP inhibitors can be exploited to treat human inflammatory diseases.

The recent studies in a variety of rodent models of experimental colitis support the role of PARP-1 activation in the pathogenesis of the disease [10-14]. PJ-34, a novel and highly potent (the in vitro IC50 is 10000 times lower than that of the prototypical compound 3-aminobenzamide) PARP-1 inhibitor, is suitable for mechanistic investigations into the regulatory roles of PARP [15]. Furthermore, PJ34 treatment improved survival in septic shock induced by bacterial peritonitis in pigs [16]. However, the role of PARP in the pathogenesis of Salmonella enteritis and the effect of the PARP-1 inhibitor PJ-34 and genetic knock down of 
PARP-1 siRNA on the inflammatory response of enterocytes to Salmonella infection are not known, prompting us to investigate the role of PJ-34 in Salmonella-induced intestinal inflammation and its mechanisms.

\section{Aim}

In this study, we aimed to examine the effect of PJ-34 on Salmonella-induced IL-6 production in Caco- 2 cells in vitro and the intracellular signaling pathways regulating the effect.

\section{Materials and Methods}

3.1. Reagents. PJ-34 was purchased from Inotek Corporation (Beverly, MA) and stock solutions made in dimethylsulfoxide (DMSO). The inhibitor was added to cells at the specified concentrations about 30-60 minutes before infection. Standard laboratory reagents were from Sigma (St. Louis, MO).

3.2. Bacterial Strains. The wild-type S. typhimurium strain SL1344 has been described previously $[17,18]$. Bacteria were grown overnight in static cultures with minimal aeration in Luria-Bertani (LB) medium. The bacteria were collected by centrifugation at $14000 \mathrm{~g}$ for 5 minutes, washed with sterile phosphate-buffered saline (PBS), and resuspended in tissue culture medium without antibiotics at a density of $4 \times 10^{9} / \mathrm{ml}$. Twenty-five $\mu \mathrm{l}$ aliquots of this suspension $\left(10^{8}\right.$ bacteria) were used to infect the cells.

3.3. Cell Culture and Infection. Caco-2 cells (ATCC, Rockville, MD), a transformed human colonic epithelial cell line, were grown in Dulbecco modified Eagle medium (DMEM) supplemented with 10\% heat-inactivated fetal calf serum, 100 units $/ \mathrm{ml}$ penicillin, $100 \mu \mathrm{g} / \mathrm{ml}$ streptomycin sulfate, and $20 \mathrm{mM}$ HEPES (Sigma) in a 5\% $\mathrm{CO}_{2}$ atmosphere at $37^{\circ} \mathrm{C}$. Passage $10-30$ was used for all experiments. For some infection experiments, cells were seeded in 12-well tissue culture plates ( $4 \mathrm{~cm}^{2} /$ well; BD Biosciences) and used at $60 \%-80 \%$ confluence.

3.4. Cell Fractionation. Cytosolic, nuclear, and membranous extracts from uninfected, infected, or PJ-34-treated Caco2 cells were prepared by the method of Wang et al. [19] with slight modifications. Cells were washed twice with icecold phosphate-buffered saline, lysed in buffer A $(10 \mathrm{mM}$ Hepes-KOH, pH 7.8, $10 \mathrm{mM} \mathrm{KCl,} 2 \mathrm{mM} \mathrm{MgCl}_{2}, 0.1 \mathrm{mM}$ EDTA, $0.1 \mathrm{mM}$ EGTA, $0.7 \%$ Nonidet P-40) with protease and phosphatase inhibitors for 30 minutes on ice, vortexed vigorously for $15 \mathrm{~s}$, and centrifuged at $3000 \times \mathrm{g}$ at $4^{\circ} \mathrm{C}$ for 10 minutes (the supernatants are the cytosolic fractions). The pelleted nuclei and membrane were resuspended in buffer B (40 mM Hepes-KOH, pH 7.8, 350 mM NaCl, 2 mM $\mathrm{MgCl}_{2}, 1 \mathrm{mM}$ EDTA, $0.2 \mathrm{mM}$ EGTA, 20\% glycerol, 1\% Nonidet P-40) with protease and phosphatase inhibitors for 60 minutes on ice, mixed vigorously for $10 \mathrm{~s}$ at 15,30 and 45 minutes, and centrifuged at $15,000 \times \mathrm{g}$ at $4^{\circ} \mathrm{C}$ for 30 minutes. Supernatants containing the nuclear proteins were stored at $-80^{\circ} \mathrm{C}$. The pelleted membrane was resuspended in lysis buffer with protease and phosphatase inhibitors. Protein concentrations in cell fractions were determined using a BioRad assay kit.

3.5. Cytokine Assay. Caco-2 monolayers were infected (apically, in the case of the polarized monolayers) in triplicate for 1 hour at $37^{\circ} \mathrm{C}$. The medium was aspirated at the end of the infection period, the cells were washed twice with sterile PBS, and medium containing gentamicin at $100 \mu \mathrm{g} / \mathrm{ml}$ was added. After incubating for 5 hours at $37^{\circ} \mathrm{C}$, the supernatant medium was collected and IL- 6 concentrations were determined by enzyme-linked immunosorbent assay (ELISA) as described below. The cells were washed with PBS and lysed with $0.2 \mathrm{ml}$ of $1 \%$ Triton X-100. An aliquot of the lysate was used to determine protein concentration by the DC protein assay (Bio-Rad, Hercules, CA) following instructions provided by the manufacturer. The IL-6 concentration was assessed using an OptEIA human IL6 enzyme-linked immunosorbent assay (ELISA) set (BD Biosciences) as described by the manufacturer. To allow comparison between multiple experiments, the amount of IL-6 produced was normalized to the protein content of the cell monolayer. Because of variations in baseline IL-6 production, the results were expressed as "fold increase", representing the normalized IL- 6 produced by infected monolayers divided by the normalized IL- 6 produced by control, uninfected monolayers.

3.6. RNA Isolation and cDNA Synthesis. Total RNA was prepared from control or infected cells with the Trizol reagent (Invitrogen Corporation, Carlsbad, CA), following the manufacturer's directions. The RNA was reversetranscribed with random hexamers using the GeneAmp kit (Roche, Nutley, NJ) as described in detail earlier $[17,18]$.

3.7. Real-Time Reverse Transcription PCR. Real-time reversetranscription PCR analyses were performed in a fluorescence temperature cycler (LightCycler; Roche Diagnostics) as described previously [20]. This technique continuously monitors the cycle-by-cycle accumulation of fluorescentlylabeled PCR product. Briefly, cDNA corresponding to $10 \mathrm{ng}$ of RNA served as a template in a $10 \mu \mathrm{l}$ reaction containing $4 \mathrm{mM} \mathrm{MgCl}_{2}, 0.5 \mu \mathrm{M}$ of each primer, and $1 \times$ LightCyclerFastStart DNA Master SYBR Green I mix (Roche Diagnostics). Samples were loaded into capillary tubes and incubated in the fluorescence thermocycler (Light-Cycler) for an initial denaturation at $95^{\circ} \mathrm{C}$ for 10 minutes followed by 45 cycles, each cycle consisting of $95^{\circ} \mathrm{C}$ for $10 \mathrm{~s}, 58^{\circ} \mathrm{C}$ for $5 \mathrm{~s}$, and $72^{\circ} \mathrm{C}$ for $20 \mathrm{~s}$. At the end of each run, melting curve profiles were produced by cooling the sample to $65^{\circ} \mathrm{C}$ for $15 \mathrm{~s}$ and then heating slowly at $0.20^{\circ} \mathrm{C} / \mathrm{s}$ up to $95^{\circ} \mathrm{C}$ with continuous measurement of fluorescence to confirm amplification of specific transcripts. Cycle-to-cycle fluorescence emission readings were monitored and analyzed using LightCycler software (Roche Diagnostics). The specificity of the amplification products was further verified by subjecting the amplification products to electrophoresis on a $2 \%$ agarose gel. The fragments were visualized by ethidium bromide staining, and 
the specificity of PCR products was verified by sequencing of representative samples. The following primers were used: IL$6,5^{\prime}$ - ATG AAC TCC TTC TCC ACA AGC GC-3' (forward primer) and 5'-G AAG AGC CCT CAG GCT GGACTG$3^{\prime}$ (reverse primer, 628bp); and glyceraldehyde-3-phosphate dehydrogenase, 5'-CCAGCCGAGCCACATCGCTC-3' (forward primer) and $5^{\prime}$-ATGAGCCCCAGCCTTCTCCAT- $3^{\prime}$. Standard curves were obtained for each primer set with serial dilutions of cDNA. All quantifications were normalized to the housekeeping gene glyceraldehyde-3-phosphate dehydrogenase. Relative expression was given as a ratio between target gene expression and glyceraldehyde-3-phosphate dehydrogenase expression.

3.8. Western Blotting. Equal amounts of total protein were separated by SDS-PAGE and then transferred to nitrocellulose membranes by semidry blotting as previously described $[17,18]$. After blocking the membranes with $5 \%$ nonfat dry milk, they were probed with antibodies to phosphorylated Akt (Cell Signaling, Beverly, MA), phosphorylated I $\kappa$ B (New England BioLabs, Beverly, MA), p65 NF- $\kappa$ B or phosphorylated ERK (Santa Cruz Biotechnology, Santa Cruz, CA), and phosphorylated JNK or p38 (New England BioLabs, Beverly, MA). The membrane was continuously incubated with appropriate secondary antibodies coupled to horseradish peroxidase and developed in the ECL Western detection reagents (Amersham-Pharmacia Biotech, Piscataway, NJ, USA). Appropriate exposures to X-ray film were made, and the filters then stripped and reprobed with antibodies to total Akt (Cell Signaling, Beverly, MA), total ERK, p38, I $\kappa \mathrm{B}, \beta$ actin, LaminA/C, or E-cadherin (Santa Cruz Biotechnology, Santa Cruz, CA) as appropriate.

3.9. Statistical Analysis. All experiments were carried out at least twice with similar results. Statistical significance was determined using Student's $t$-test.

\section{Results}

4.1. PJ-34, PARP-1 Inhibitor, Potentiates Salmonella-Induced IL-6 Production. In Figure 1, we found that enhancement of the IL-6, either in protein secretion or mRNA expression, was increased as higher concentration of PJ-34 was applied (statistically significant after $20 \mu \mathrm{M}$ PJ-34). Then, we proceeded to study the mechanisms of the effect of PJ-34 on IL-6.

\subsection{PJ-34 Enhances and Prolongs the Activation of NF- $\kappa B$} by Increasing I $\kappa B-\alpha$ Degradation and Nuclear Translocation of $N F-\kappa B$. To further elucidate the mechanism by which PJ-34, PARP-1 inhibitor, increased Salmonella-induced IL6 production in intestinal epithelial cells, we examined the intracellular signaling pathways that have been implicated in expression of the cytokine. The transcriptional regulation of the IL-6 gene is complex and involves different transcription factors [21]. Transcription factor NF- $\kappa \mathrm{B}$ is a key transcription factor in the regulation of cytokines and chemokines, including IL-6 gene expression [22]. Activation of the NF$\kappa \mathrm{B}$ pathway, as well as the mitogen-activated protein kinases

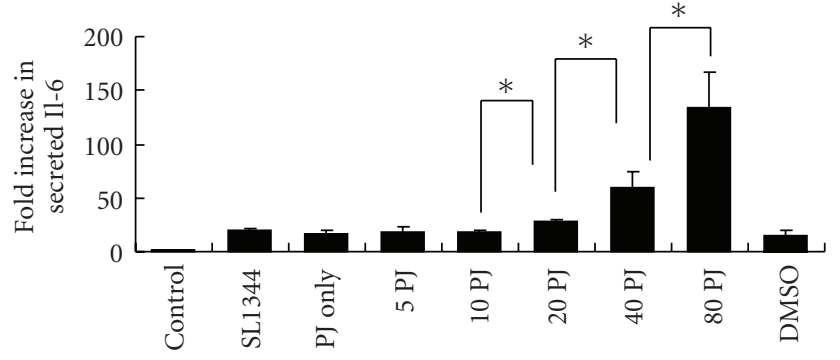

(a)

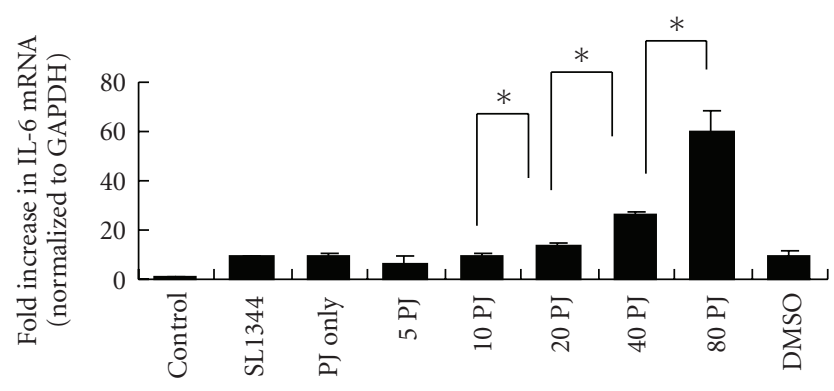

(b)

Figure 1: (a) Effect of PJ-34 on Salmonella-induced IL-6 protein secretion. Caco-2 cells were left untreated (control), or treated with $5,10,20,40$, or $80 \mu \mathrm{M} \mathrm{PJ}-34(5,10,20,40,80 \mathrm{PJ})$, or with a volume of DMSO (D) equivalent to the highest concentration of PJ-34. They were then uninfected (control and PJ only) or infected with the wild-type $S$. typhimurium strain SL1344 for 1 hour. Supernatant was analyzed by ELISA 6 hours later for IL-6. The amount of IL- 6 produced is shown as the fold increase over uninfected, control cells. The results are representative of the results of two similar experiments. The data are the means \pm standard deviations for three determinations. ${ }^{*} P<.05$. (b) Effect of PJ-34 on Salmonella-induced IL-6 mRNA. Caco-2 cells were left untreated, or treated with $5,10,20,40$, or $80 \mu \mathrm{M}$ PJ-34. They were then uninfected or infected with the wild-type $S$. Typhimurium strain SL1344 for 1 hour. Total RNA was prepared 3 hours later and analyzed by real-time quantitative PCR to estimate amounts of IL-6 transcript. The amount of IL- 6 mRNA produced, normalized to the corresponding amount of GAPDH transcript, is shown as the fold increase over uninfected, control cells. The results are representative of the results of two similar experiments. The data are the means \pm standard deviations for three determinations. ${ }^{*} P<.05$.

(MAPK); extracellular growth factor-regulated kinase (ERK) and p38, has all been shown to be involved in Salmonellainduced cytokines and chemokines production $[17,23,24]$. To determine the involvement of these signals in the effect of PJ-34 on Salmonella-infected intestinal epithelial cells, Caco-2 cells were left untreated, or treated with $40 \mu \mathrm{M} \mathrm{PJ}-$ 34 , and then infected with the wild-type Salmonella strain SL1344. Activation of the NF- $\kappa$ B pathway was assessed by examining nuclear translocation of NF- $\kappa \mathrm{B}$ and degradation of the inhibitor protein I $\kappa$ B- $\alpha$. As shown in Figure 2(a), Salmonella infection resulted in degradation of $\mathrm{I} \kappa \mathrm{B}-\alpha$ and nuclear translocation of NF- $\kappa \mathrm{B}$. PJ-34 enhances and prolongs the activation of NF- $\kappa \mathrm{B}$ by increasing I $\kappa \mathrm{B}-\alpha$ degradation and nuclear translocation of NF- $\kappa$ B (Figure 2(b)), resulting in subsequent upregulation of IL-6 gene transcription. 


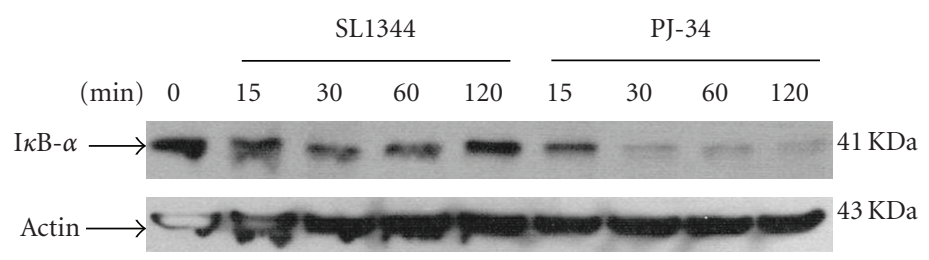

(a)

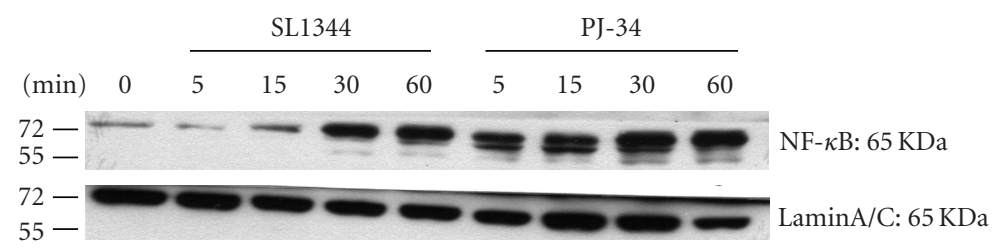

(b)

Figure 2: Effect of PJ-34 on Salmonella-induced activation of NF- $\kappa B$. NF- $\kappa B$ is a central regulator of the intestinal epithelial cell innate immune response induced by infection with enteroinvasive bacteria. The Western blots illustrate (a) the expression of I $\kappa$-B $\alpha$ proteins in cytosolic extracts of Caco-2 cells exposed to wild-type S. Typhimurium strain SL1344 in the absence or presence of PJ-34. Actin works as a normalization of nuclear protein and (b) the expression of p65 NF-kB proteins in nuclear extracts of Caco-2 cells exposed to wild-type S. Typhimurium strain SL1344 in the absence or presence of PJ-34. Actin and LaminA/C work as a normalization of cytosolic and nuclear protein, respectively. The results shown are representative of 3 separate experiments.

4.3. PJ-34 Enhanced ERK Phosphorylation in SalmonellaInfected Intestinal Epithelial Cells but Did Not Alter the JNK orp38-MAPK Phosphorylation. We also examined activation of MAPKs (ERK, JNK, and p38 kinases) (Figure 3), using antibodies specific to either the phosphorylated (activated) or total forms of these proteins. While inhibition of PARP with PJ-34 had no effect on Salmonella-dependent phosphorylation of the p38 kinase and JNK, it had a clear and reproducible enhancing effect on activation of the ERK kinase. These findings suggest that MAPK pathways are involved in the regulatory effect of PJ-34 on Salmonella-induced IL6 production in intestinal epithelial cells. However, they differentially regulate the cell responses.

4.4. PJ-34 Has No Effect on the Membranous Recruitment and Activation of AKT in Salmonella-Infected Caco-2 Cells. $\mathrm{NF}-\kappa \mathrm{B}$ is a key transcription factor in the regulation of proinflammatory cytokines and chemokines. Besides, an intimate relationship between that NF- $\kappa \mathrm{B}$ and PARP- 1 is demonstrated by the multiple lines of evidence showing that the synthesis of poly(ADP-ribose) promotes NF- $\kappa \mathrm{B}$ transactivation and inhibition of PARP-1 can attenuate this activation and subsequent cytokine expression. Some reports have also demonstrated that PARP inhibitors induced the phosphorylation and activation of Akt in lipopolysaccharidetreated mice or cultured cells during oxidative stress, raising the protective effect of PARP inhibition mediated through the PI3K-kinase/Akt pathway. In our previous studies, we demonstrated NF- $\kappa \mathrm{B}$ and PI3K/Akt pathways play important roles in the pathogenesis of Salmonella enteritis [17, 18]. However, we found inhibition of PARP-1 with PJ-34 had no effect on Salmonella-induced phosphorylation of Akt (Figure 4).

\section{Discussion}

Our study gave first insight into the regulatory effect on the inflammatory responses by a novel PARP-1 inhibitor PJ-34 in Salmonella-infected intestinal epithelial Caco-2 cells. In this study, we found that wild-type $S$. typhimurium induced IL-6 production in Caco- 2 cells, whereas PJ-34 enhanced IL6 expression, either secreted IL-6 or IL-6 mRNA. Although commonly considered a proinflammatory cytokine [3], there is also evidence that IL-6 has important antiinflammatory properties and may exert protective effects in various tissues [4-6]. The studies from Hasselgren et al. suggest that IL6 produced by enterocytes may have antiinflammatory and cell-protective effects and that increased IL-6 levels in gut mucosa may counteract some of the injurious effects of sepsis and endotoxemia $[25,26]$. Intense inflammatory response induced by Salmonella infection results in destruction of the epithelial layer of the intestinal mucosa that may lead to translocation of bacteria and absorption of endotoxins into the circulation $[27,28]$. Consequently, translocation of bacteria and absorption of endotoxins may have profound systemic effects and may result in bacteremia as well as endotoxemia. However, IL-6 has been described to be a mediator of epithelial barrier protection [29] and endogenous IL-6 plays an essential, nonredundant role in limiting intestinal injury and cell death [30]. Probiotic bacterium L. paracasei may exert some of their beneficial effects by enhancing IL6 production in enterocytes subjected to an inflammatory stimulus [31].

In our study, PJ-34 upregulated the Salmonella-induced IL-6 production. That might explain the marked beneficial effect of PJ-34 in various models of local inflammation in rodents, in which the levels of IL-6 were not measured [32]. PJ-34 may provide the antiinflammatory and protective 


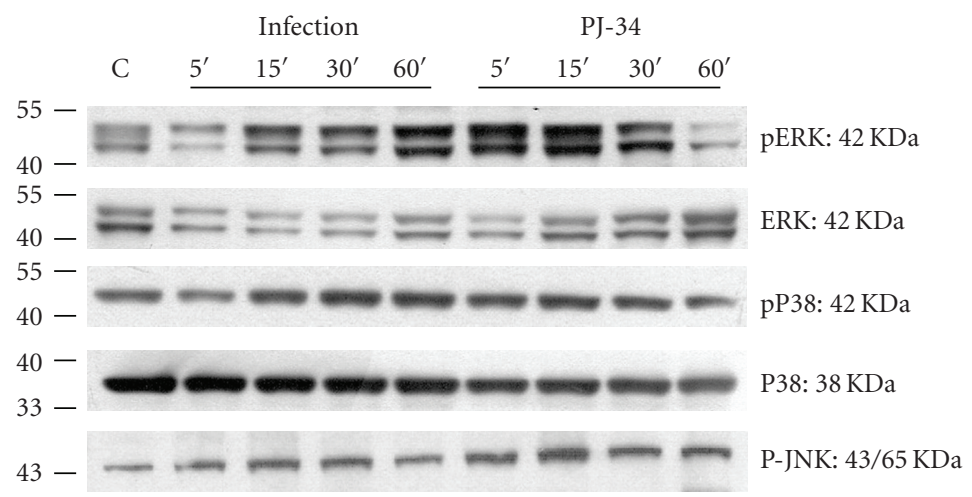

FIGURE 3: Effect of PJ-34 on Salmonella-activated intracellular signals. Caco-2 cells were left untreated, or treated with $40 \mu \mathrm{M}$ PJ-34, and then infected with wild-type S. Typhimurium strain SL1344 for the times indicated. Activations of the ERK, JNK, and p38 were analyzed in whole cell protein by immunoblotting with antibodies to phosphorylated (p) ERK, JNK, and p38 and total ERK and p38. The activation of ERK was enhanced by PJ-34 but not the JNK and p38. The results shown are representative of 3 separate experiments.

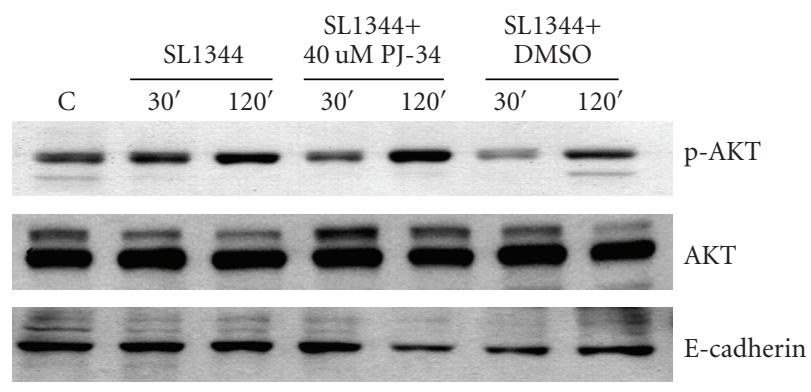

FIGURE 4: Effect of PJ-34 on Salmonella-activated intracellular signals. Caco-2 cells were left untreated, or treated with $40 \mu \mathrm{M}$ PJ-34, and then infected with wild-type $S$. Typhimurium strain SL1344 for the times indicated. Activation of the AKT pathway was analyzed in cell fraction (membrane part) by immunoblotting with antibodies to phosphorylated (p) AKT and total AKT. E-cadherin works as a normalization of membranous protein. The results shown are representative of 3 separate experiments. The activation and recruitment of Akt were not significantly suppressed by PJ-34.

effects on intestinal epithelial cells to counteract the invasion and injurious effects of Salmonella endotoxemia through the upregulation of enterocyte IL- 6 production. To the best of our knowledge, up to now, no report has demonstrated that PJ-34 upregulated Salmonella-induced IL-6 expression in intestinal epithelial cells.

It has been previously reported that genetic deficiency or pharmacological inhibition of PARP-1 confers beneficial effects in experimental models of colitis [10-14, 32, 33]. Blockade of PARP inhibits intercellular adhesion molecule 1 (ICAM-1) or cyclooxygenase-2 expression [13, 33], neutrophil recruitment $[13,14,33]$, oxidant generation, and mucosal injury in murine colitis. However, one (rare) report has demonstrated the effect of PJ-34 on Il-6 production [34]. Analysis of local expression of the IL- 6 in skeletal muscle after ischemia and $48 \mathrm{~h}$ of reperfusion showed significantly higher levels in the PJ-34 treated group when compared with saline. The authors suggested that not all cytokine activity during reperfusion is deleterious. It might provide protective or antiinflammatory effects.
The transcriptional regulation of the IL- 6 gene is complex and involves different transcription factors [21]. Transcription factor $\mathrm{NF}-\kappa \mathrm{B}$ is a central regulator of IL6 gene expression [22]. Activation of the NF- $\kappa \mathrm{B}$ pathway, as well as the mitogen-activated protein kinases (MAPKs): extracellular growth factor-regulated kinase (ERK) and p38, has been shown to be involved in Salmonella-induced cytokines and chemokines production [17, 22, 23]. Besides, the diversity of signal pathways involved in the protective effect of PARP inhibitors depends on the experiment models and inhibitors used. Most studies [10-14] have shown reduced activation of transcription factors NF- $\kappa$ B and AP-1, while very few studies have shown increased phosphorylation of MAPK and PI3K/Akt pathways except JNK. L-2286, a novel PARP inhibitor, facilitated the ischemia-reperfusioninduced activation of Akt, ERK, and p38-MAPK in both isolated hearts and in vivo cardiac injury [35].

To further elucidate the mechanism by which PJ-34 upregulated IL-6 production, we examined various signaling pathways that have been implicated in expression of the cytokine. While inhibition of PARP-1 with PJ-34 had no effect on Salmonella-induced phosphorylation of the p38 kinase, JNK, or Akt, it had a clear and reproducible enhancing effect on activation of the ERK kinase and nuclear translocation of NF- $\kappa \mathrm{B}$. These findings are in contrast with previous reports in A549 lung epithelial cells [36] or WRL68 human liver cells [37]. They found that PJ-34 suppressed NF- $\kappa$ B activation but not AP- 1 in cytokine-stimulated A549 cells and had no effect on the expression of most chemokines [36]. They also showed that PJ-34 had no effect on phosphorylation of all MAPKs. In WRL-68 cells transfected with PARP siRNA or pharmacologically inhibited by PJ-34, the phosphorylation of Akt (Ser ${ }^{473}$ ) increased during oxidative stress compared with wild type. Nevertheless, in accordance with our results, Kameoka et al. [38] demonstrated an inverse correlation between PARP and NF- $\kappa \mathrm{B}$ activities. They showed that $\mathrm{Cl}-3527$ cells with the lowest PARP content expressed 35 -fold greater activity of NF- $\kappa$ B than wild-type L1210 cells. However, a discrepancy seems to exist with Cl3527 and the PARP-1-gene disrupted cells [39, 40], which 
showed markedly suppressed NF- $\kappa \mathrm{B}$-dependent signaling. These authors explained that the discrepancy may be due to the difference in the residual poly(ADP-ribosylating) activity in these mutants. It suggested that transcription factors and signal pathways varied between different cell types and may also vary within the same cell depending on stimulus [41].

Our results suggest that might also modulate a diverse array of signaling cascades beside gene expression. To our knowledge, this is the first in vitro report, which attributes a critical role to NF- $\kappa \mathrm{B}$ and ERK in the Salmonella-induced upregulation of IL- 6 in Caco-2 cells, conferred by PARP-1 inhibitor PJ-34.

\section{Conclusions}

In conclusion, the present study provides the first evidence that PJ-34 may enhance IL-6 production in enterocytes subjected to Salmonella infection and that the effect of PJ-34 is, at least in part, through NF- $\kappa \mathrm{B}$ and ERK signal pathways. Because other studies have shown that IL-6 has antiinflammatory and protective effects in the intestinal mucosa, the present results offer a novel mechanism by which PJ-34 may exert some of its beneficial effects, although additional in vivo experiments will be needed to define the role of IL- 6 in cell-protective effects provided by PJ-34 treatment.

\section{Acknowledgments}

The authors wish to thank Bobby Cherayil for his numerous productive discussions. This work was supported in part by National Science Community Grant no. NSC- 95-2314-B182A-197.

\section{References}

[1] L. Eckmann and M. F. Kagnoff, "Intestinal mucosal responses to microbial infection," Springer Seminars in Immunopathology, vol. 27, no. 2, pp. 181-196, 2005.

[2] D. A. Papanicolaou, R. L. Wilder, S. C. Manolagas, and G. P. Chrousos, "The pathophysiologic roles of interleukin-6 in human disease," Annals of Internal Medicine, vol. 128, no. 2, pp. 127-137, 1998.

[3] M. R. Pinsky, J.-L. Vincent, J. Deviere, M. Alegre, R. J. Kahn, and E. Dupont, "Serum cytokine levels in human septic shock: relation to multiple-system organ failure and mortality," Chest, vol. 103, no. 2, pp. 565-575, 1993.

[4] T. P. Shanley, J. L. Foreback, D. G. Remick, T. R. Ulich, S. L. Kunkel, and P. A. Ward, "Regulatory effects of interleukin-6 in immunoglobulin G immune-complex-induced lung injury," American Journal of Pathology, vol. 151, no. 1, pp. 193-203, 1997.

[5] Z. Xing, J. Gauldie, G. Cox, et al., "IL-6 is an antiinflammatory cytokine required for controlling local or systemic acute inflammatory responses," Journal of Clinical Investigation, vol. 101, no. 2, pp. 311-320, 1998.

[6] B. E. Barton and J. V. Jackson, "Protective role of interleukin 6 in the lipopolysaccharide-galactosamine septic shock model," Infection and Immunity, vol. 61, no. 4, pp. 1496-1499, 1993.
[7] H. C. Jung, L. Eckmann, S.-K. Yang, et al., "A distinct array of proinflammatory cytokines is expressed in human colon epithelial cells in response to bacterial invasion," Journal of Clinical Investigation, vol. 95, no. 1, pp. 55-65, 1995.

[8] D. N. Baldwin, V. Vanchinathan, P. O. Brown, and J. A. Theriot, "A gene-expression program reflecting the innate immune response of cultured intestinal epithelial cells to infection by Listeria monocytogenes," Genome Biology, vol. 4, no. 1, article R2, 2003.

[9] G. Haskó, J. G. Mabley, Z. H. Németh, P. Pacher, E. A. Deitch, and C. Szabó, "Poly(ADP-ribose) polymerase is a regulator of chemokine production: relevance for the pathogenesis of shock and inflammation," Molecular Medicine, vol. 8, no. 5, pp. 283-289, 2002.

[10] E. Mazzon, L. Dugo, J.-H. Li, et al., "GPI 6150, a PARP inhibitor, reduces the colon injury caused by dinitrobenzene sulfonic acid in the rat," Biochemical Pharmacology, vol. 64, no. 2, pp. 327-337, 2002.

[11] H. B. Jijon, T. Churchill, D. Malfair, et al., "Inhibition of poly(ADP-ribose) polymerase attenuates inflammation in a model of chronic colitis," American Journal of Physiology, vol. 279, no. 3, pp. G641-G651, 2000.

[12] B. Zingarelli, P. W. Hake, T. J. Burroughs, G. Piraino, M. O'Connor, and A. Denenberg, "Activator protein-1 signalling pathway and apoptosis are modulated by poly(ADP-ribose) polymerase-1 in experimental colitis," Immunology, vol. 113, no. 4, pp. 509-517, 2004.

[13] S. Sánchez-Fidalgo, I. Villegas, A. Martín, M. SánchezHidalgo, and C. Alarcón de la Lastra, "PARP inhibition reduces acute colonic inflammation in rats," European Journal of Pharmacology, vol. 563, no. 1-3, pp. 216-223, 2007.

[14] C. Szabó, L. H. K. Lim, S. Cuzzocrea, et al., "Inhibition of poly (ADP-ribose) synthetase attenuates neutrophil recruitment and exerts antiinflammatory effects," Journal of Experimental Medicine, vol. 186, no. 7, pp. 1041-1049, 1997.

[15] F. G. Soriano, L. Virág, P. Jagtap, et al., "Diabetic endothelial dysfunction: the role of poly(ADP-ribose) polymerase activation," Nature Medicine, vol. 7, no. 1, pp. 108-113, 2001.

[16] R. D. Goldfarb, A. Marton, E. Szabo, et al., "Protective effect of a novel, potent inhibitor of poly(adenosine 5' -diphosphateribose) synthetase in a porcine model of severe bacterial sepsis," Critical Care Medicine, vol. 30, no. 5, pp. 974-980, 2002.

[17] F.-C. Huang, A. Werne, Q. Li, E. E. Galyov, W. A. Walker, and B. J. Cherayil, "Cooperative interactions between flagellin and SopE2 in the epithelial interleukin-8 response to Salmonella enterica serovar typhimurium infection," Infection and Immunity, vol. 72, no. 9, pp. 5052-5062, 2004.

[18] F.-C. Huang, Q. Li, and B. J. Cherayil, "A phosphatidylinositol-3-kinase-dependent anti-inflammatory pathway activated by Salmonella in epithelial cells," FEMS Microbiology Letters, vol. 243, no. 1, pp. 265-270, 2005.

[19] F.-S. Wang, C.-J. Wang, H.-J. Huang, H. Chung, R.-F. Chen, and K. D. Yang, "Physical shock wave mediates membrane hyperpolarization and Ras activation for osteogenesis in human bone marrow stromal cells," Biochemical and Biophysical Research Communications, vol. 287, no. 3, pp. 648-655, 2001.

[20] J. Harder, U. Meyer-Hoffert, K. Wehkamp, L. Schwichtenberg, and J.-M. Schröder, "Differential gene induction of human $\beta$ defensins (hBD-1, -2, -3, and -4) in keratinocytes is inhibited by retinoic acid," Journal of Investigative Dermatology, vol. 123, no. 3, pp. 522-529, 2004. 
[21] W. Vanden Berghe, L. Vermeulen, G. De Wilde, K. De Bosscher, E. Boone, and G. Haegeman, "Signal transduction by tumor necrosis factor and gene regulation of the inflammatory cytokine interleukin-6," Biochemical Pharmacology, vol. 60, no. 8, pp. 1185-1195, 2000.

[22] A. A. Parikh, A. L. Salzman, C. D. Kane, J. E. Fischer, and P.-O. Hasselgren, "IL-6 production in human intestinal epithelial cells following stimulation with IL- $1 \beta$ is associated with activation of the transcription factor NF- $\kappa \mathrm{B}$," Journal of Surgical Research, vol. 69, no. 1, pp. 139-144, 1997.

[23] T. A. Libermann and D. Baltimore, "Activation of interleukin6 gene expression through the NF- $\kappa \mathrm{B}$ transcription factor," Molecular and Cellular Biology, vol. 10, no. 5, pp. 2327-2334, 1990.

[24] M. Vitiello, M. D’Isanto, E. Finamore, R. Ciarcia, A. Kampanaraki, and M. Galdiero, "Role of mitogen-activated protein kinases in the iNOS production and cytokine secretion by Salmonella enterica serovar Typhimurium porins," Cytokine, vol. 41, no. 3, pp. 279-285, 2008.

[25] A. A. Parikh, M. R. Moon, C. D. Kane, A. L. Salzman, J. E. Fischer, and P.-O. Hasselgren, "Interleukin-6 production in human intestinal epithelial cells increases in association with the heat shock response," Journal of Surgical Research, vol. 77, no. 1, pp. 40-44, 1998.

[26] Q. Wang, X. Sun, T. A. Pritts, H. R. Wong, and P.-O. Hasselgren, "Induction of the stress response increases interleukin-6 production in the intestinal mucosa of endotoxaemic mice," Clinical Science, vol. 99, no. 6, pp. 489-496, 2000.

[27] J. W. Ding, R. Andersson, V. Soltesz, R. Willén, and S. Bengmark, "Obstructive jaundice impairs reticuloendothelial function and promotes bacterial translocation in the rat," Journal of Surgical Research, vol. 57, no. 2, pp. 238-245, 1994.

[28] A. S. Taner, I. Cinel, I. Ozer, U. Onde, D. Taner, and C. Koksoy, "Poly(ADP-ribose) synthetase inhibition reduces bacterial translocation in rats after endotoxin challenge," Shock, vol. 16, no. 2, pp. 159-162, 2001.

[29] L. Wang, S. Srinivasan, A. L. Theiss, D. Merlin, and S. V. Sitaraman, "Interleukin-6 induces keratin expression in intestinal epithelial cells: potential role of keratin-8 in interleukin-6induced barrier function alterations," Journal of Biological Chemistry, vol. 282, no. 11, pp. 8219-8227, 2007.

[30] X. Jin, T. A. Zimmers, Z. Zhang, R. H. Pierce, and L. G. Koniaris, "Interleukin-6 is an important in vivo inhibitor of intestinal epithelial cell death in mice," to appear in Gut.

[31] N. Reilly, V. Poylin, M. Menconi, A. Onderdonk, S. Bengmark, and P.-O. Hasselgren, "Probiotics potentiate IL-6 production in IL- $1 \beta$-treated Caco- 2 cells through a heat shock-dependent mechanism," American Journal of Physiology, vol. 293, no. 3, pp. R1169-R1179, 2007.

[32] J. G. Mabley, P. Jagtap, M. Perretti, et al., "Anti-inflammatory effects of a novel, potent inhibitor of poly(ADP-ribose) polymerase," Inflammation Research, vol. 50, no. 11, pp. 561569, 2001.

[33] B. Zingarelli, C. Szabo, and A. L. Salzman, "Blockade of poly(ADP-ribose) synthetase inhibits neutrophil recruitment, oxidant generation, and mucosal injury in murine colitis," Gastroenterology, vol. 116, no. 2, pp. 335-345, 1999.

[34] M. F. Conrad, H. Albadawi, D. H. Stone, R. S. Crawford, F. Entabi, and M. T. Watkins, "Local administration of the poly ADP-ribose polymerase (PARP) inhibitor, PJ34 during hindlimb ischemia modulates skeletal muscle reperfusion injury," Journal of Surgical Research, vol. 135, no. 2, pp. 233 $237,2006$.
[35] A. Pálfi, A. Tóth, G. Kulcsár, et al., "The role of Akt and mitogen-activated protein kinase systems in the protective effect of poly(ADP-ribose) polymerase inhibition in langendorff perfused and in isoproterenol-damaged rat hearts," Journal of Pharmacology and Experimental Therapeutics, vol. 315, no. 1, pp. 273-282, 2005.

[36] K. Erdèlyi, A. Kiss, E. Bakondi, et al., "Gallotannin inhibits the expression of chemokines and inflammatory cytokines in A549 cells," Molecular Pharmacology, vol. 68, no. 3, pp. 895904, 2005.

[37] A. Tapodi, B. Debreceni, K. Hanto, et al., "Pivotal role of Akt activation in mitochondrial protection and cell survival by poly(ADP-ribose)polymerase-1 inhibition in oxidative stress," Journal of Biological Chemistry, vol. 280, no. 42, pp. 3576735775, 2005.

[38] M. Kameoka, K. Ota, T. Tetsuka, et al., "Evidence for regulation of NF- $\kappa$ B by poly(ADP-ribose) polymerase," Biochemical Journal, vol. 346, part 3, pp. 641-649, 2000.

[39] F. J. Oliver, J. Ménissier-de Murcia, C. Nacci, et al., "Resistance to endotoxic shock as a consequence of defective NF- $\kappa \mathrm{B}$ activation in poly (ADP-ribose) polymerase-1 deficient mice," The EMBO Journal, vol. 18, no. 16, pp. 4446-4454, 1999.

[40] P. O. Hassa and M. O. Hottiger, "A role of poly (ADP-Ribose) polymerase in NF- $\kappa \mathrm{B}$ transcriptional activation," Biological Chemistry, vol. 380, no. 7-8, pp. 953-959, 1999.

[41] D. D. Hershko, B. W. Robb, G. Luo, and P.-O. Hasselgren, "Multiple transcription factors regulating the IL-6 gene are activated by cAMP in cultured Caco- 2 cells," American Journal of Physiology, vol. 283, no. 5, pp. R1140-R1148, 2002. 


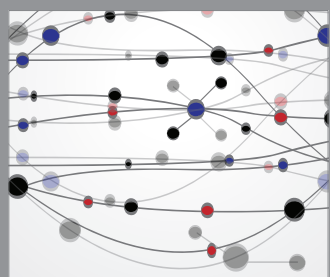

The Scientific World Journal
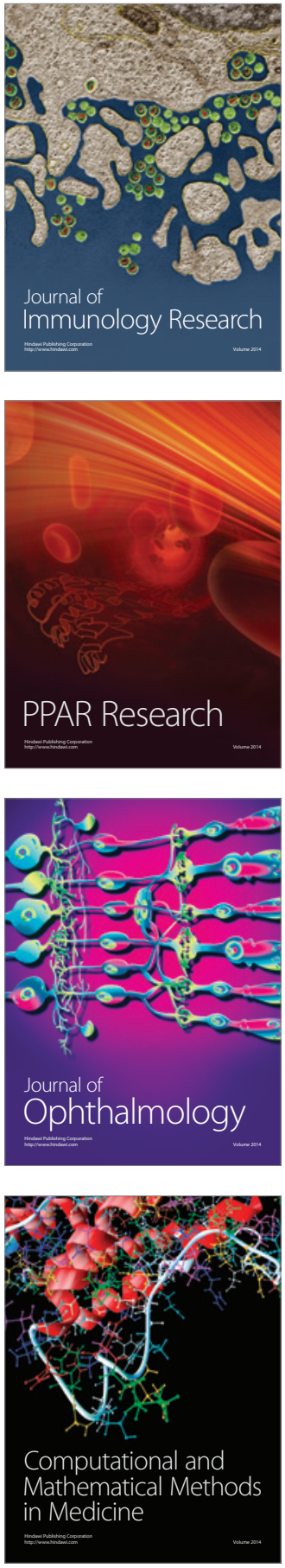

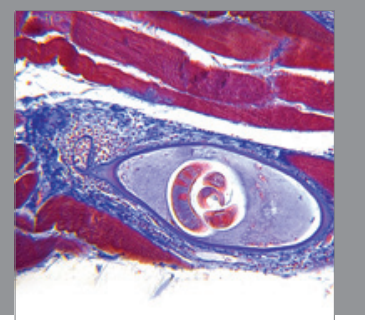

Gastroenterology

Research and Practice
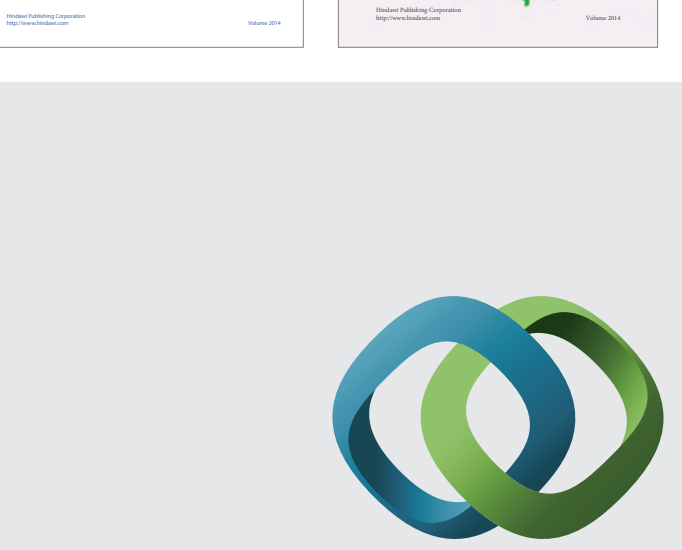

\section{Hindawi}

Submit your manuscripts at

http://www.hindawi.com
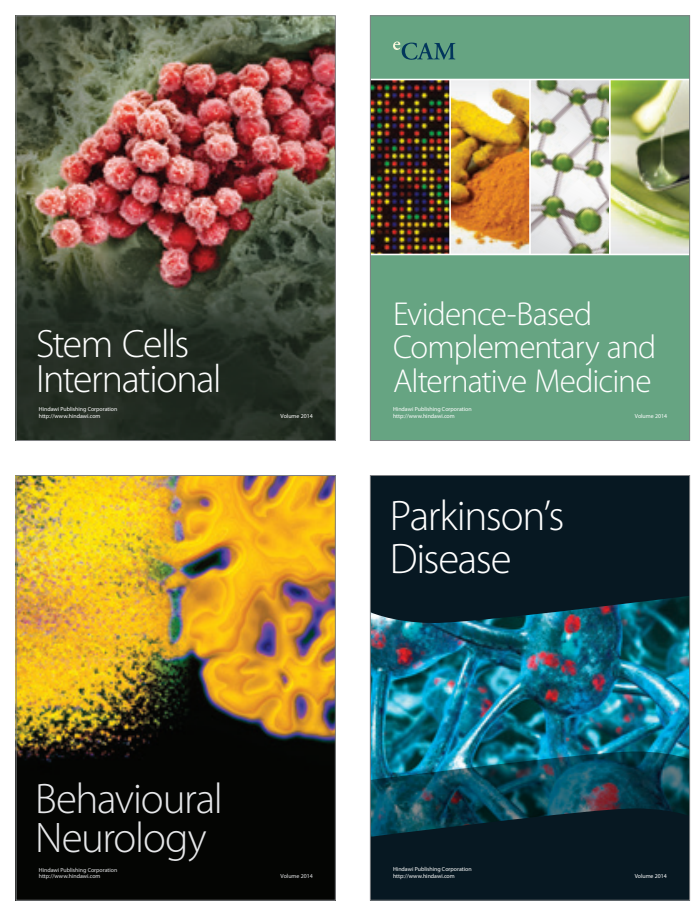

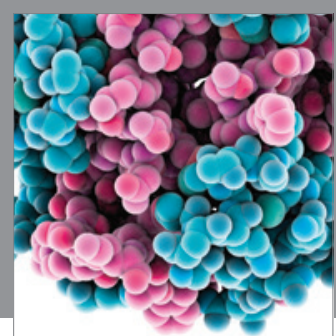

Journal of
Diabetes Research

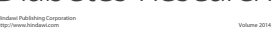

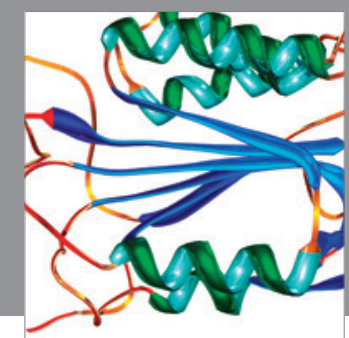

Disease Markers
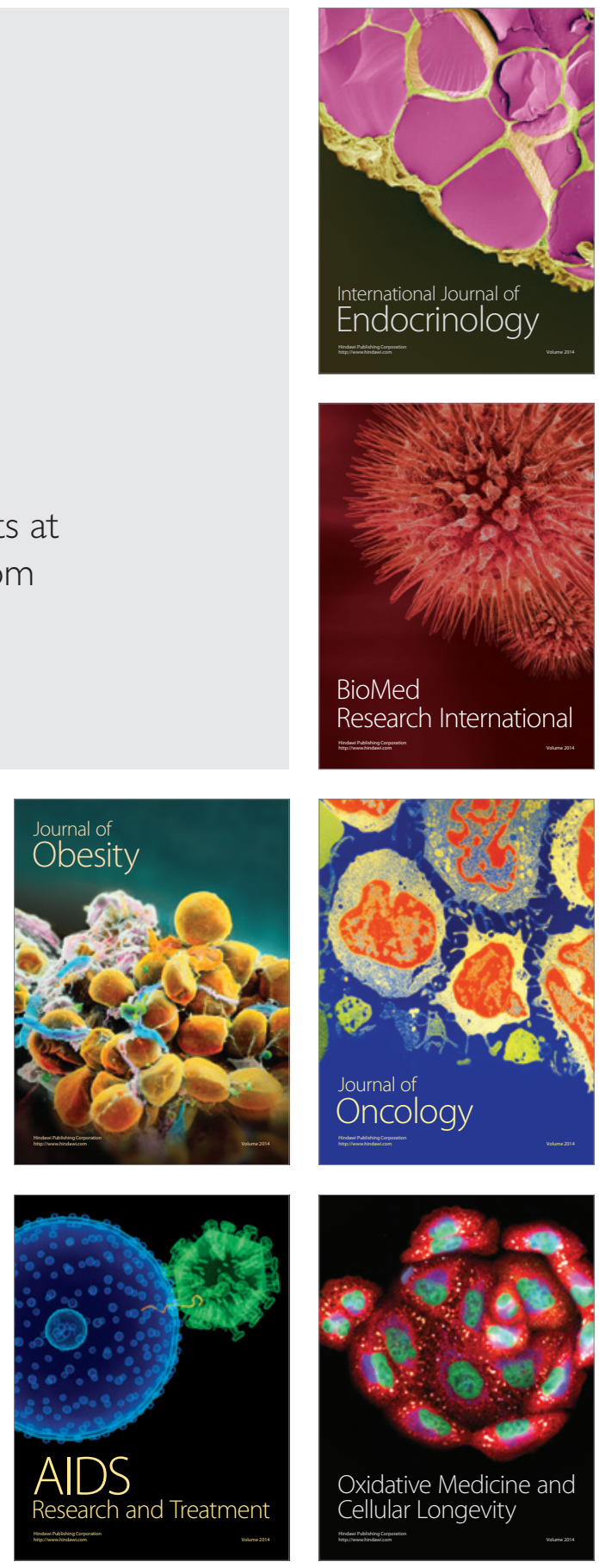